\title{
Triglyceride to High Density Lipoprotein Cholesterol Ratio in Acute Myocardial Infarction And Its Relationship with Angiographic Severity
}

\author{
Md. Sahadat Hossain ${ }^{1}$, Prabir Kumar Das ${ }^{1}$, Syed Ali Ahsan², Biplob Bhattacharjee ${ }^{1}$, Anisul Awal ${ }^{1}$, Sandipan Das ${ }^{1}$, \\ Iqbal Mahmud ${ }^{1}$, Sukanta Dhar ${ }^{1}$, A.B.K. Bashiruddin ${ }^{1}$, Partha Bhattacharyya ${ }^{1}$, Debabrata Bhattacharya ${ }^{1}$.
}

\begin{abstract}
:
Background: In acute myocardial infarction (AMI) the extent of the coronary artery lesion is evaluated by coronary angiogram (CAG). Recent evidences suggest that, ratio of triglyceride and high-density lipoprotein cholesterol (TG/HDL-C) could be a non-invasive marker for the prediction of the extent of coronary artery lesion. The aim of this study was to evaluate the association between TG/HDL-C ratio and the extent of coronary artery lesion assessed by coronary CAG among AMI patients.

Objective: The aim of this study was to assess relationship between TG to HDL-C ratio and extent of coronary artery lesion in AMI patients.

Materials \& Method: This cross-sectional study was carried out in the Department of Cardiology, Chittagong Medical College Hospital in 224 admitted AMI patients. Blood samples were taken within 24 hours of admission following AMI for fasting lipid profile assessment, (total cholesterol (TC), HDL-C, low-density lipoprotein cholesterol (LDL-C) and TG). Each patient was underwent CAG within 2 to 6 weeks of the events and angiographic findings were classified according to presence of
\end{abstract}

significant stenosis, number of vessel involved and Gensini score.

Results: The subjects consisted of 197 males and 27 females with a mean age of $51.24( \pm 11.22)$ years. Mean value of TC was $185.74( \pm 41.96) \mathrm{mg} / \mathrm{dL}$, TG was 222.17 $( \pm 99.05) \mathrm{mg} / \mathrm{dL}$, HDL-C was $38.92( \pm 5.46) \mathrm{mg} / \mathrm{dL}$, LDL-C was $127.99( \pm 36.94) \mathrm{mg} / \mathrm{dL}, \mathrm{TG} / \mathrm{HDL}-\mathrm{C}$ was 5.91 ( \pm 2.99$)$, median Gensini score was 28 (Range:1-146). Analysis of receiver operating characteristic curves showed that only TG/HDLC and TG were useful for detecting high Gensini score (score $>42$ ), with the former more area under the curve (AUC: $0.611 ; 95 \% \mathrm{Cl}: 0.531-0.691 ; p=0.008$ ). The TG/HDL-C was an independent predictive factor (Odds ratio: $2.706 ; 95 \% \mathrm{Cl}$ : 1.397-5.242; $p=0.003$ ) for the presence of significant coronary artery lesion on CAG. Linear regression analysis revealed that, age and TG/HDL-C ratio significantly predicted Gensini score.

Conclusion: TG and HDL-C ratio was independently associated with extent of coronary artery lesion.

Key words: TG/HDL-C ratio, AMI, Angiographic Severity,

(Bangladesh Heart Journal 2021; 36(2): 82-88)

Introduction:

Around the world, cardiovascular disease (CVD) is recognized as the leading cause of death (accounting for approximately $31 \%$ of all deaths) and is predicted to remain as such in 2030. ${ }^{1}$ Myocardial infarction (MI) is one of the most common form of CVD. ${ }^{2}$ World Health Organization forecasts an increase of $11 \%$ in the burden

1. Department of Cardiology, Chattogram Medical College,

2. Department of Cardiology, Bangabandhu Sheikh Mujib Medical University, Dhaka.

Address of Correspondence: Dr. Sahadat Hossain, Department of Cardiology, Chattogram Medical College, Chattogram. E-mail: sagar.somc42@gmail.com

DOI: https://doi.org/10.3329/bhj.v36i2.56033

Copyright $\odot 2017$ Bangladesh Cardiac Society. Published by Bangladesh Cardiac Society. This is an Open Access articles published under the Creative Commons Attribution-NonCommercial 4.0 International License (CC BY-NC). This license permits use, distribution and reproduction in any medium, provided the original work is properly cited and is not used for commercial purposes. 
of CVD by 2030, bringing the worldwide number of MI and stroke to approximately 36.2 million. $^{3}$

Although the incidence of $\mathrm{MI}$ is decreased in the industrialized nations partly because of improved health systems and implementation of effective public health strategies, nevertheless the rates are surging in the developing countries such as South Asia, parts of Latin America, and Eastern Europe.4 Due to lack of national population-based surveys or central administrative health data it is hard to find accurate information on the prevalence of $\mathrm{MI}$ in Bangladesh. However, a recent review observed a high CVD prevalence along with an upward trend in Bangladeshi adults. ${ }^{5}$

"Dyslipidemia" refers to an abnormality within the lipid profile, encompassing a variety of disorders relating to elevations in total cholesterol (TC), low density lipoprotein cholesterol (LDL-C), or triglyceride (TG), or conversely, lower levels of high-density lipoprotein cholesterol (HDLC). The dyslipidemia may present as a single disorder affecting only one lipoprotein parameter, or may represent a combination of lipoprotein abnormalities, such as elevated TG and low HDL-C. ${ }^{6}$

The third Adult Treatment Panel (ATP) Guidelines of the US National Cholesterol Education Program (NCEP 2001) recommend a full fasting lipoprotein profile, including TG, TC, HDL-C, and LDL-C. According to ATP III guideline dyslipidemia is considered with Serum TC > $200 \mathrm{mg} / \mathrm{dl}$, TG >150 mg/dl, LDL-C >100 mg/dl, HDL-C $<40 \mathrm{mg} / \mathrm{dl}$ (male) and $<50 \mathrm{mg} / \mathrm{dl}$ (female). Although the guidelines only provide for evaluation of individual lipid fractions, the application of ratios such as TC/HDL-C and TG/HDL-C may offer a refined risk assessment by simultaneously considering both anti-atherogenic and atherogenic lipid parameters.

Selective coronary angiography remains the gold standard to determine the extent of CAD, because it is the only technique that can simultaneously provide both functional and anatomic information for the estimation of ischemic burden of CAD. ${ }^{7}$

Significant CAD was defined as $\geq 70 \%$ stenosis in any of the three major epicardial coronary arteries or a left main coronary artery stenosis $\geq 50 \%$. Patients were grouped as having single vessel disease (SVD), double vessel disease (DVD) and triple vessel disease (TVD) according to the number of vessel involvement. ${ }^{8}$

To determine the severity of CAD Gensini score was also estimated in the study. Gensini score is a widely used angiographic scoring system for quantifying the severity of CAD. ${ }^{9}$
Several studies have reported the condition of dyslipidemia among CAD patients of Bangladesh but the study assessing association between TG to HDL-C ratio and severity of CAG findings among MI patients. ${ }^{10-11}$

There are few studies on TG/HDL-C ratio in AMI and their Angiographic correlation in Bangladesh. The relevance of this study is to correlate relationship of TG/HDL-C in $\mathrm{AMI}$ and to see their angiographic severity. In developing countries like Bangladesh, coronary angiogram facility is limited to the tertiary care hospitals and specialized hospital. If we can find any relation between TG/HDL-C ratio and severe coronary artery lesion, then that will helpful to influence the decision of a strategy non invasive investigation in patients with AMI. So we can predict invasive investigation by non-invasive way, which will in long run reduce morbidity and mortality due to coronary artery disease in our country.

\section{Materials and Methods:}

Study design and patients:

This is a hospital based Cross-sectional study was conducted on 224 patients of AMI admitted in Department of cardiology, Chittagong Medical College \& Hospital, Chattogram, Bangladesh and underwent CAG within 26 weeks of events during May 2019 to April 2020.

Purposive sampling was done. Patients were excluded on the background of refusal to give consent, severe comorbid condition like renal failure, liver failure, stroke.

\section{Study procedure:}

In this study, patients with acute myocardial infarction were assessed during their admission for the eligibility in the study. After inclusion an informed written consent was taken from the patient. Demographic profile of the patient including age, sex and occupation and major risk factors like diabetes, hypertension, dyslipidemia, smoking, family history of CAD, were recorded. The body height was measured in the standing position without shoes. Weight was measured similarly without shoes and heavy dresses.

With all aseptic precautions $5 \mathrm{ml}$ of fasting blood sample was drawn and sent to biochemistry laboratory for analysis. Fasting lipid profile was determined on the day of blood collection in enzymatic kinetic method by Siemens Dimension EXL 200 auto analyzer made in Germany. Then TG/HDL-C ratio was calculated as more than 4 and less than 4.

Subsequently patients were undergoing coronary angiography after the AMI events as per the hospital protocol. Coronary Angiography was performed in Cath 
lab of cardiology ward by SHIMADZU BRANSIST alexa C12 (ceiling mounted angiographic machine) made in Japan. Coronary angiogram was obtained for each coronary vessel in e"2 projections in cardiology ward. Analysis of the coronary angiograms was performed visually by an experienced operator. The severity of the CAD was assessed by vessel score and Gensini score.

\section{Data analysis:}

The statistical analysis was carried out by using Statistical Package for Social Sciences (SPSS-23). Quantitative or continuous variables were described as mean \pm standard deviation median (range). Means were compared using independent sample t-test between two groups. Qualitative or categorical variables were described as frequencies and proportions. Proportions were compared by using chi-square test.

Correlation between two variables was determined by Pearson correlation coefficient. A ROC curve was used to determine the diagnostic performance of TG/ HDL-C ratio in the prediction of the extension of coronary artery lesion. Binary logistic regression and linear regression analysis were conducted to determine the independent predictive factors for severe CAG findings. Theses analysis included the variables which were found to have significant association with CAG findings in bivariate analysis. Statistical significance and confidence interval were set at $p<0.05$ and $95 \%$ level respectively.

\section{Results:}

The present cross sectional study intended to investigate the relation of TG to HDL- $C$ ratio with angiographic severity of CAD in patient's admitted with AMI. Finally, 224 patients admitted with AMI and had CAG were included in the analysis. The findings obtained from data analysis are presented below:

The mean age of 224 patients with AMI was $51.24 \pm 11.22$ (Range: $26-70$ ) years and $87.9 \%$ of participants were men. Regarding occupation majority of the men were either doing institutional service or un-institutional job and in women majority were housewife.

In the 224 AMI patients most prevalent risk factor was dyslipidemia present in $211(94.2 \%)$ of patients followed by smoking in 174 (77.8\%), overweight and obesity in $131(59 \%)$, DM in $82(37 \%)$, hypertension in $73(33 \%)$ and family history of CAD in $59(26 \%)$ of patients (Figure 1).

Out of 224 patients with AMI 148 of them were diagnosed as NSTEMI (66.1\%) and other $76(33.9 \%)$ were diagnosed as STEMI (Figure 2).

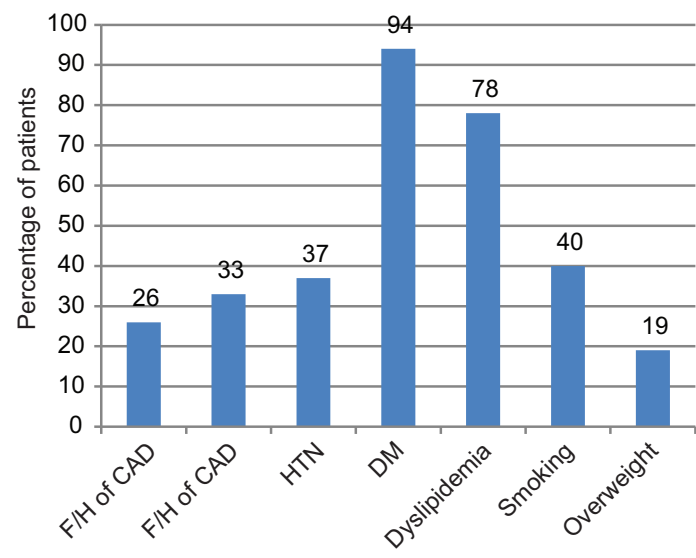

Fig.-1: Distribution of risk factors of CAD among AMI patients $(n=224)$

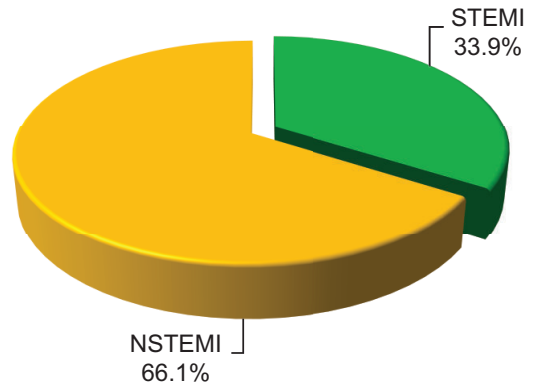

Fig.-2: Distribution of the patients by AMI type $(n=224)$

Table-I

Lipid profile of the patients $(n=224)$

\begin{tabular}{ll}
\hline Parameters & Mean $( \pm$ SD $)$ \\
\hline Total cholesterol $(\mathrm{mg} / \mathrm{dl})$ & $185.74 \pm 41.96$ \\
LDL cholesterol $(\mathrm{mg} / \mathrm{dl})$ & $127.99 \pm 36.94$ \\
HDL cholesterol $(\mathrm{mg} / \mathrm{dl})$ & $38.92 \pm 5.46$ \\
Triglyceride $(\mathrm{mg} / \mathrm{dl})$ & $222.17 \pm 99.05$ \\
Triglyceride/HDL-C & $5.91 \pm 2.99$ \\
\hline
\end{tabular}

Table I shows that, mean LDL-C, and mean TG values were higher than the expected normal values $(<100 \mathrm{mg} /$ $\mathrm{dl}$ and $<150 \mathrm{mg} / \mathrm{dl}$ respectively) among the studied patients. Similarly mean HDL-C level was lower than the expected normal values ( $\geq 40 \mathrm{mg} / \mathrm{dl}$ for male and $\geq 50 \mathrm{mg} /$ $\mathrm{dl}$ for female). However, mean TC value was within the expected normal range $(<200 \mathrm{mg} / \mathrm{dl})$ in the studied AMI patients. Mean TG/HDL-C ratio was also higher than the normal expected value $<4$.

Among the lipid sub fractions most prevalent lipid abnormality was high LDL-C present in $183(81.7 \%)$ of patients followed by high TG present in 163 (72.8\%), low 
HDL-C in 163 (56.7\%) and high TC in 80 (35.7\%) patients (Figure 3). About 63\% (141/224) patients had TG/HDL-C ratio $\geq 4$. Only $13(5.8 \%)$ patients had normal lipid profile in all sub fractions and had TG/HDL-C ratio $<4$.

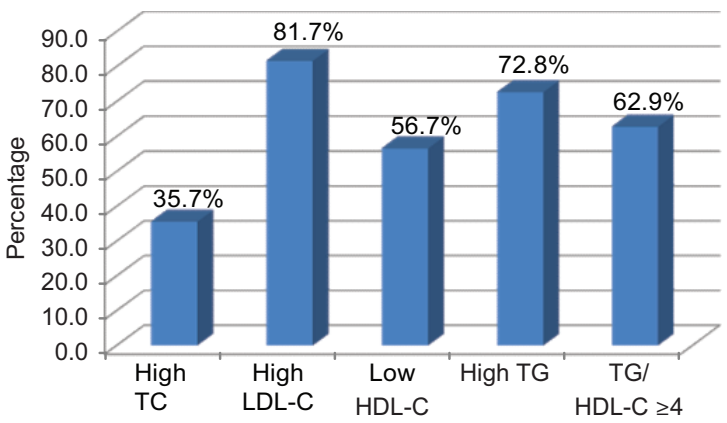

Fig.-3: Prevalence of abnormal lipid sub-fractions and ratio among $A M I$ patients $(n=224)$

Table-II

Angiographic profile of the patients $(n=224)$

\begin{tabular}{llc}
\hline Characteristics & & Frequency (\%) \\
\hline Significant CAD & Present & $153(68.3)$ \\
& Absent & $71(31.7)$ \\
No of involved vessel & Single vessel & $110(49.1)$ \\
& Double vessel & $55(24.6)$ \\
& Triple vessel & $59(26.3)$ \\
& Median (Range) & $28(1-146)$ \\
Gensini score & Low (score $\leq 20)$ & $45(33.5)$ \\
& Intermediate & $78(34.8)$ \\
& (score 21-42) & $71(31.7)$ \\
\hline
\end{tabular}

Data are expressed as frequency (percentage) if not otherwise mentioned.

Out of 224 AMI patients underwent CAG, majority (68.3\%) have significant obstruction in angiogram. About half of the patient's had SVD. About one fourth of the patients had double vessel and triple vessel diseases each. Patients' were divided into tertile according to their Gensini score, and 45 (33.5\%) patients were in lowest tertile of Gensini score had score equal to or below 20 and $71(31.7 \%)$ patients were in highest tertile had score $>42$ (Table II).
Table-III

Bivariate association between CAG findings and TG/ $H D L-C$ category in AMI patients $(n=224)$

\begin{tabular}{|c|c|c|c|}
\hline \multirow[b]{2}{*}{ Variables } & \multicolumn{2}{|c|}{ TG/HDL-C of the patients } & \multirow[b]{2}{*}{$p$ value } \\
\hline & $\begin{array}{c}<4 \\
(n=83)\end{array}$ & $\begin{array}{c}\geq 4 \\
(n=141)\end{array}$ & \\
\hline \multicolumn{4}{|c|}{ Significant CAD } \\
\hline $\begin{array}{l}\text { Present } \\
\text { Absent }\end{array}$ & $\begin{array}{l}40(48.2) \\
43(51.8)\end{array}$ & $\begin{array}{l}113(61.7) \\
28(38.3)\end{array}$ & $0.001^{*}$ \\
\hline \multicolumn{4}{|c|}{$\begin{array}{l}\text { Number of vessel } \\
\text { involved }\end{array}$} \\
\hline Single & $50(60.2)$ & $60(42.6)$ & \\
\hline Double & $18(21.7)$ & $37(26.2)$ & $0.027^{*}$ \\
\hline Triple & $15(18.1)$ & $44(31.2)$ & \\
\hline \multicolumn{4}{|l|}{ Gensini tertile } \\
\hline Low & $32(38.6)$ & $41(29.1)$ & \\
\hline Medium & $35(42.2)$ & $48(34.0)$ & $0.021 *$ \\
\hline High & $16(19.3)$ & $52(36.9)$ & \\
\hline
\end{tabular}

$\mathrm{a}_{<}<40 \mathrm{mg} / \mathrm{dl}$ for male and $<50 \mathrm{mg} / \mathrm{dl}$ for female. Data are expressed as frequency (percentage); ${ }^{*} p$ value derived from Chi-square test. Significant values are in bold face.

Table III shows that association between angiographic findings of the AMI patients and their TG/HDL-C pattern. It shows that, patients with TG/HDL-C $\geq 4$ have severe CAG findings in terms of all the three characteristics like, significant stenosis, vessel number and Gensini tertile compared to the patients with TG/HDL-C $<4$.

The scattered plot in Figure 4 shows that, among the 224 AMI patient's TG/HDL-C had a positive correlation with Gensini score. It indicates that, as the TG/HDL-C ratio increases the Gensini score of the AMI patients also increase. This positive correlation was statistically significant $(p=0.011)$.

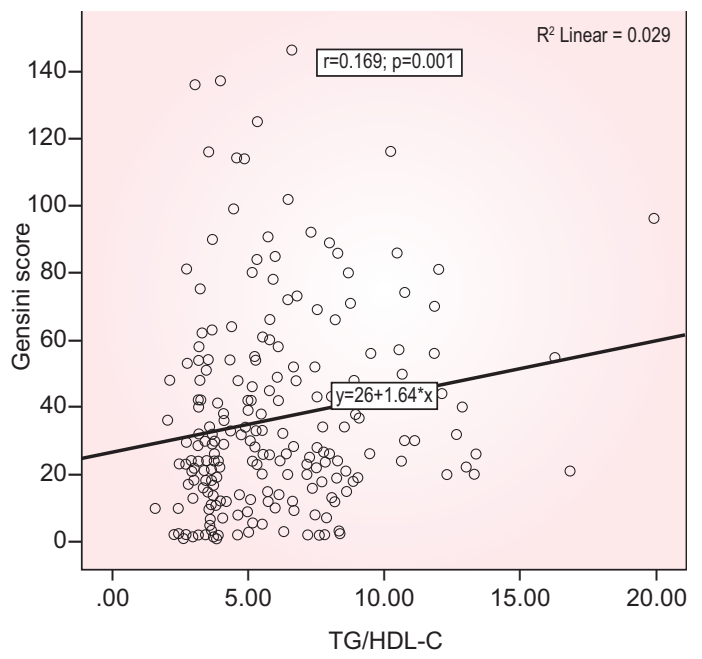

Fig.-4: The relationship between TG/HDL-C and Gensini scores $(n=224)$ 


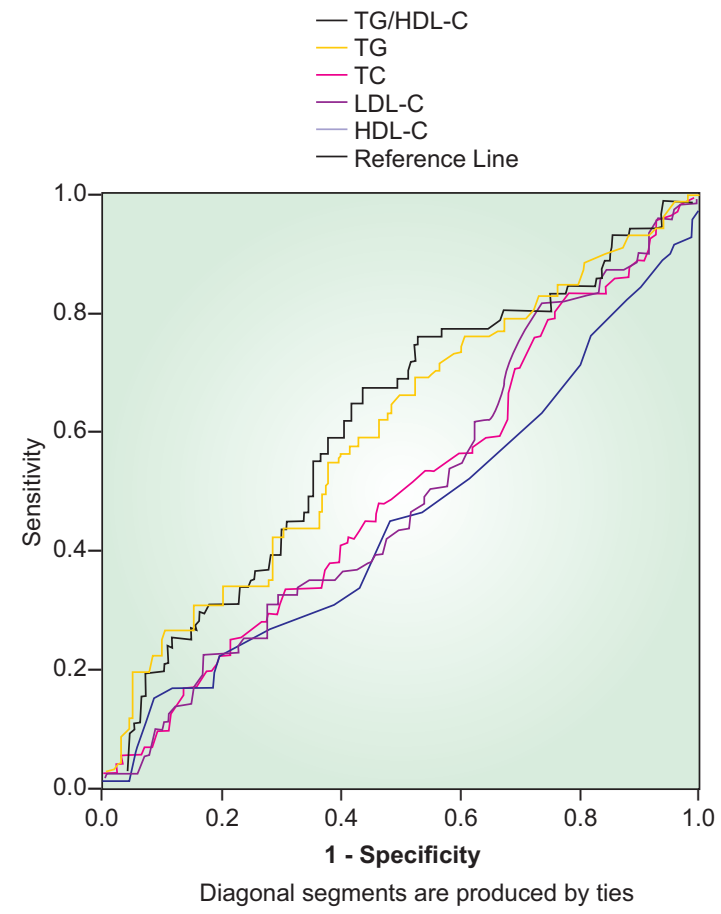

Fig.-5: $R O C$ curves for $T G / H D L-C$ ratio and individual lipid sub-fractions in predicting for high Gensini score (>42) in AMI patients ( $n=224)$.

Table-IV

Area under the curve (AUC) with 95\% CI for different lipid parameters for predicting high Gensini score $(n=224)$

\begin{tabular}{lcccc}
\hline Variables & AUC & \multicolumn{2}{c}{$95 \%$ Cl of AUC } & $p$ value \\
& & Lower & $\begin{array}{c}\text { Upper } \\
\text { limit }\end{array}$ & limit \\
\hline TG/HDL-C & 0.611 & 0.531 & 0.691 & 0.008 \\
TG & 0.600 & 0.519 & 0.680 & 0.016 \\
TC & 0.503 & 0.421 & 0.585 & 0.946 \\
LDL-C & 0.496 & 0.415 & 0.577 & 0.919 \\
HDL-C & 0.455 & 0.370 & 0.539 & 0.274 \\
\hline
\end{tabular}

$\mathrm{Cl}$ : confidence interval

ROC analysis demonstrated a significant diagnostic value of TG/HDL-C ratio and TG level of the AMI patients to detect AMI patients having high Gensini score (Figure 5 and Table IV).

\section{Discussion:}

The present study observed the mean age of the AMI patients was $51.24 \pm 11.22$ years ranging from 26 to 70 years. Previous studies from Bangladesh reported similar age distribution of patients with AMI with mean age around 51 years with maximum number of the patients in the age range of $51-60$ years. ${ }^{10-12}$ In contrast to these findings from Bangladesh, studies from India reported comparatively higher mean age like $62.74 \pm$ 13.6 years, $1356.06 \pm 11.29$ years, 14 and $56.75 \pm 10.47$ years. 15 Moreover, western study has reported higher mean age as compared to these studies conducted in Asia such as study (68 years). ${ }^{16}$

With regard to sex distribution, $87.9 \%$ of our study population was male and only $12.1 \%$ was female (male: female ratio of $7.2: 1$ ), showing a clear male preponderance. Similar sex ratio with male predominance were also observed by other studies. ${ }^{10-12}$

Regarding the traditional risk factors, it was found in the current study that, most of the patients of AMI (211/224; $94.2 \%$ ) had unrecognized dyslipidemia. Similar to this finding reported such high prevalence $(85.1 \%$ and $82.1 \%$ respectively) of unrecognized dyslipidemia among the admitted patients for first time AMI. ${ }^{12,17}$ Followed by dyslipidemia the other risk factors in descending order were smoking $(77.8 \%)$, overweight and obesity $(59 \%)$, DM (37\%), hypertension (33\%) and family history of CAD (26\%). Amin et al., (2014) reported that smoking was found in the highest (65.3\%) number followed by hypertension (51.7\%), overweight (50.8\%), DM (42.4\%) and family history of CAD (10.2\%). Major CAD risk factors were noted to be very prevalent in the present study, which is similar to previous published data evaluating the prevalence of these risk factors in Bangladesh. ${ }^{18-19}$

The present study demonstrated that, among the lipid sub fractions most prevalent lipid abnormality was high LDL-C present $(81.7 \%)$, followed by high TG $(72.8 \%)$, low HDL-C (56.7\%) and high TC (35.7\%). Several studies have reported varying prevalence and type of dyslipidemia. The prevalence of high TG (28-72.2\%), high LDL cholesterol (23.3-44.5\%), low HDL (27-72.2\%) and high TC (19-38.7\%) were observed from different region of India and Bangladesh. 10,11,21 These variations can be explained by differences in the study population with respect to age and sex distribution, inclusion of patients with CVD and population or hospital-based study.

In the current study coronary angiographic severity was assessed by presence or absence of significant obstruction, number of vessel involvement and Gensini score calculation. It was observed that $68.3 \%$ patients had significant obstruction.

Regarding vessel involvement $49.1 \%$ had SVD, $24.6 \%$ DVD and $26.3 \%$ TVD. Almost similar result has been observed by other study. ${ }^{14,16}$

This study showed that patients with TG/HDL-C $\geq 4$ have severe CAG findings in terms of all the three parameters 
like, significant stenosis, vessel number and Gensini tertile compared to the patients with TG/HDL-C $<4$. About $38.3 \%$ patients without significant obstruction had high TG/HDL-C ratio $(\geq 4)$ whereas $61.7 \%$ patients with significant obstruction had high TG/HDL-C ratio $(\geq 4)$.

The multivariate analysis by binary logistic regression showed that a subject with TG/HDL-C value $\geq 4$ had higher odds of having significant coronary lesion than AMI patients with TG/HDL-C vale $<4$ (adjusted $\mathrm{OR}=2.706$; $95 \% \mathrm{Cl}=1.397-5.242)$.

Previous reports have shown that high TG/HDL-C ratios correlate independently with presence of angiographic coronary artery disease (defined as stenosis $>70 \%$ ) among men and women even after adjustment for traditional risk factors, including diabetes. 22-24 The present study was also able to reproduce this finding in AMI patients and extend the observation to demonstrate that the TG/HDL-C ratio was also associated with coronary artery disease severity as expressed by a Gensini score.

\section{Conclusion:}

In this study high TG/HDL-C ratio $(\geq 4)$ correlated with severity of CAD in terms of Gensini score and significant obstruction. High TG/HDL-C ratio was found as the most important predictor of severe coronary heart disease among all the lipid variables examined.

\section{References:}

1. Mathers CD, Loncar D. Projections of global mortality and burden of disease from 2002 to 2030. PLoS medicine.2006; 3(11):442.

2. World Health Organization. Global Status Report on Noncommunicable Diseases 2014. 2015; Available from: https://apps.who.int/iris/bitstream/ handle/10665/148114/97892 41564854.

3. World Health Organization. Global atlas on cardiovascular disease prevention and control. World Health Organization; World Heart Federation; World Stroke Organization. 2011; Available: http:// www.who.int/cardiovascular_diseases/publication s/atlas_cvd/en/

4. Laslett LJ, Alagona P, Clark BA, Drozda, JP, Saldivar F, Wilson S R et al. The Worldwide Environment of Cardiovascular Disease: Prevalence, Diagnosis, Therapy, and Policy Issues. Journal of the American College of Cardiology.2012; 60 (25): S1-S49.

5. Chowdhury M, Haque MA, Farhana Z, Anik, AM, Chowdhury $\mathrm{A} \mathrm{H}$, Haque $\mathrm{S} \mathrm{M}$, et al. Prevalence of cardiovascular disease among Bangladeshi adult population: a systematic review and meta-analysis of the studies. Vascular health and risk management.2018; (14):165-181.

6. Kori JK, Gerg B. Understanding the Essentials of Blood Lipid Metabolism. 2007. Available from: https://www.medscape.com/viewarticle/451762_1

7. Roxana Mehran, George D Dangas. Coronary angiography and intravascular imaging. Braunwald's heart diseases.2019;19(20):374.

8. Neeland I J, Patel RS, Eshtehardi P, Dhawan S, McDaniel MC, Rab S T, et al. Coronary angiographic scoring systems: an evaluation of their equivalence and validity. American heart journal. 2012;16(44): 547-552.

9. Gensini G G. A more meaningful scoring system for determining the severity of coronary heart disease.The American journal of cardiology. 1983;51(3):606 (Online). https://doi.org/10.1016/ s0002-9149(83)80105-2

10. Alam MZ, Rahman A, Hoque S, Irfan S, Miah MB, Hossain MS. Pattern of Dyslipidemia in different type of Myocardial Infarction.Bangladesh Critical Care Journal.2017; 5(2):106-109.

11. Amin MR, Rahman MA, Alam N, Hasan MN, Hasan GMS. Relationship between triglyceride HDLcholesterol ratio and severity of coronary artery disease in patient with acute coronary syndrome. Bangladesh Med journal. 2014; 43(3):157-161.

12. Chowdhury BR, Das J, Islam HMN, Ahmed FU. Conventional risk factors and lipid profiles in firsttime acute myocardial infarction patients in Chattogram, Bangladesh.BGC Trust Medical College Journal. 2019;14(2):5-9.

13. Patel A, Vishwanathan S, Nair T, Bahuleyan, CG, Jayaprakash VL. Sex Differences in the Presentation, Diagnosis, and Management of Acute Coronary Syndromes: Findings from the KeralaIndia ACS Registry. Glob Heart. 2015;10(4):273-80.

14. Sidhu NS, Rangaiah S, Ramesh D, Veerappa K, Manjunath CN.Clinical Characteristics, Management Strategies, and In-Hospital Outcomes of Acute Coronary Syndrome in a Low Socioeconomic Status Cohort: An Observational Study from Urban India,Clinical Medicine Insights. Cardiology.2020; 14(1179546820918897). (online). https://doi.org/10.1177/1179546820918897 
15. Singh SS, Paul SK, Pal R, Thatkar PV. Acute coronary syndrome-related mortality audit in a teaching hospital at Port Blair, India. J Family Med Prim Care. 2017; 6(3):502-508.

16. Penalva RA, Huoya M, Correia LC, Feitosa GS, Ladeia AM. Lipid profile and intensity of atherosclerosis disease in acute coronary syndrome. Arquivos brasileiros de cardiologia. 2008; 90(1):24-30.

17. Gaziano JM, Hennekens CH, O'Donnell CJ, Breslow JL, Buring JE. Fasting triglycerides, highdensity lipoprotein, and risk of myocardial infarction. Circulation.1997;96(8):2520 2525.

18. Ahmed MI, Mohammad Akhtaruzzaman, K Rahman MA, Mahmod MS, Nahar S. Relationship between HDL-Cholesterol and Angiographic Severity of Coronary Artery Disease. Bangladesh Heart Journal.2018; 33(1): 32-38.

19. Das PK, Kamal SM, Murshed M. Acute myocardial infarction in young Bangladeshis: A comparison with older patients. Journal of Indian college of cardiology.2015;5(1):20-24
20. Islam M, Bhattacharjee B, Chowdhury MA, Siddique A, Karim AM. Outcome of Acute Myocardial Infarction Patients Admitted in a Tertiary Care Hospital. Medicine Today. 2017;28(1):6-8.

21. Joshi $P$, Islam S, Pais P, Reddy S, Dorairaj P, Kazmi $\mathrm{K}$, et al. Risk Factors for Early Myocardial Infarction in South Asians Compared with Individuals in Other Countries. JAMA. 2007;297(3): 286-294.

22. Frohlich, J, Dobiásová, M. Fractional Esterification Rate of Cholesterol and Ratio of Triglycerides to HDL-Cholesterol Are Powerful Predictors of Positive Findings on Coronary Angiography.Clinical Chemistry. 2003;49(11):1873-1880.

23. Bittner V, Johnson BD, Zineh I, Rogers W J, Vido $D$, Marroquin OC, et al. The triglyceride/high density lipoprotein cholesterol ratio predicts all- cause mortality in women with suspected myocardial infarction. American heart journal.2009; 157(3): 548-555.

24. Jeppesen, J, Hein H O, Suadicani P, Gyntelberg F. Low Triglycerides- High High-Density Lipoprotein Cholesterol and Risk of Ischemic Heart Disease.Archives of Internal Medicine. 2001;161(3): 361-366. 